\title{
In vivo study of the effects of exogenous hydrogen sulfide on lung mitochondria in acute lung injury in rats
}

Quansheng Du ${ }^{1,2}$, Chao Wang ${ }^{3}$, Nan Zhang ${ }^{4}$, Guofeng Li $i^{5}$, Meng Zhang ${ }^{6}$, Liping Li $i^{5}$, Qingzeng Zhang ${ }^{5}$ and Jianxin Zhang ${ }^{1^{*}}$

\begin{abstract}
Background: Acute lung injury (ALI) is a serious disease with high incidence in ICU, and impaired mitochondria function plays a significant role in ALI. In this study, we examined the possible roles of exogenous hydrogen sulfide $\left(\mathrm{H}_{2} \mathrm{~S}\right)$ in lung mitochondria regulation in $\mathrm{ALI}$ rats.

Methods: The rat ALI model was induced by an intra-tongue vein Lipopolysaccharide (LPS) injection. We used sodium hydrosulphide (NaHS) as the $\mathrm{H}_{2} \mathrm{~S}$ donor. We randomly divided 40 Sprague-Dawley rats into five groups: control, LPS injury, LPS + low-dose NaHS $\left(0.78 \mathrm{mg} \cdot \mathrm{kg}^{-1}\right)$, LPS + middle-dose NaHS $\left(1.56 \mathrm{mg} \cdot \mathrm{kg}^{-1}\right)$, and LPS + high-dose NaHS $\left(3.12 \mathrm{mg} \cdot \mathrm{kg}^{-1}\right)$. Rats were killed $3 \mathrm{~h}$ after NaHS administration. We calculated a semi-quantitative histological index of lung injury assessments and measured the lung wet-to-dry weight ratio. We further analyzed serum for interleukin-1 $\beta$ levels using enzyme-linked immunosorbent assays. We observed lung mitochondria ultrastructures with an electron microscope. We examined oxidative stress markers in lung mitochondria and the mitochondrial swelling and activity. We analyzed lung mitochondria and cytosol Cyt-c protein expression using Western blotting.

Results: Compared to the control group, the quantitative assessment score index, wet-to-dry weight ratios, and interleukin-1 $\beta$ content in the LPS injury group were significantly increased and the mitochondrial ultrastructure damaged. Furthermore, mitochondrial activity, adenosine triphosphatease, superoxide dismutase, glutathione peroxidase, and mitochondrial Cyt-c protein expression were significantly decreased, and malondialdehyde content, mitochondrial swelling, and cytosol Cyt-c protein expression were significantly increased in the LPS injury group compared to the control group. These effects were lessened by NaHS.
\end{abstract}

Conclusion: Exogenous $\mathrm{H}_{2} \mathrm{~S}$ provided a protective effect against ALI by decreasing the mitochondrial lipid peroxidation level and protecting the cell structure in the LPS-induced rat models. Its regulatory effect on lung mitochondria is positively correlated with the dosage.

Keywords: Acute lung injury, Mitochondria, Lipopolysaccharide, Exogenous hydrogen sulfide, Mitochondrial lipid peroxidation

\footnotetext{
* Correspondence: zhangjx100@163.com

'Department of Pharmacology, Hebei Medical University, 361 Zhongshan Eastern Road, Shijiazhuang 050017, China

Full list of author information is available at the end of the article
} 


\section{Background}

Acute lung injury (ALI) and its most severe manifestation, acute respiratory distress syndrome (ARDS), is a clinical syndrome characterized by acute hypoxemic respiratory failure, bilateral pulmonary infiltrates on frontal chest radiograph consistent with edema, and normal cardiac filling pressures [1]. The resulting lung damage can evoke lung failure and multiple organ dysfunctions associated with increased mortality [2]. Sepsis (the presence of pus-forming bacteria or their toxins in the blood or tissues) is one of the most important ALI/ARDS causes [3]. Lipopolysaccharide (LPS), a major gram-negative bacillary endotoxin component, plays an important role in initiating inflammatory response and causing systemic inflammatory response syndrome (SIRS) and sepsis. The lung is one of the target organs primarily injured by endotoxin infection and sepsis. ALI induced by LPS is an acute pulmonary inflammation response in the lung, in which the accumulation and activation of polymophonuclear neutrophil (PMN) and oxygen free radical release are the key links [4]. LPS injection in vivo is a classic method to manufacture a sepsis-induced animal ALI model [5]. Inflammatory cell activation and increased oxidative stress are implicated in this pathogenesis [6]. Malondialdehyde [MDA], an end-product of membrane lipid peroxidation, adenosine triphosphatease [ATPase], anti-oxidants superoxide dismutase [SOD], and glutathione peroxidase [GPx] are currently considered the basic oxidative stress markers.

LPS damages the mitochondrial structure, and the ATP enzyme and oxidative phosphorylation coupling process leads to energy metabolism disorders. LPS-induced-ALI can cause abnormal mitochondrial structures and functions, and abnormalities tend to change other cell organelles and the whole cell, thereby increasing ALI's degree [7]. Studies have shown that oxidant-induced death and dysfunction of pulmonary vascular cells play important roles in ALI's evolution, and oxygen radicals that damage DNA in their mitochondria play an important role in its process [8]. A cell's energy sub-units mitochondria are significant to biological energy metabolism. When subjected to outside influences, its dysfunction may be an important lung injury component. Mitochondrial dysfunction may be a main cause for ALI, so improving mitochondrial function may be important for treating ALI.

In recent years, hydrogen sulfide $\left(\mathrm{H}_{2} \mathrm{~S}\right)$ has been seen as very important in a wide range of physiological and pathological functions [9-13]. Recently, some experiments confirmed that endogenous $\mathrm{H}_{2} \mathrm{~S}$ played an important role in inflammatory disease pathogenesis and associated organ injury, such as acute pancreatitis [14], sepsis [15], ischemia/ reperfusion injury [16], and lung injury (ventilator-induced lung injury [17], or oleic acid-induced ALI [18]), succeeded in exerting organ protective effects. Some experiments have shown that $\mathrm{H}_{2} \mathrm{~S}$ may participate in ALI/ARDS pathogenesis. However, few experiments focus on exogenous $\mathrm{H}_{2} \mathrm{~S}$ (inhalational or parenteral administration) $[2,18,19]$, so $\mathrm{H}_{2} \mathrm{~S}$-related therapy for ALI/ARDS may be a potential therapeutic approach [20]. Also, injecting NaHS (exogenous $\mathrm{H}_{2} \mathrm{~S}$ ) in normal rats directly results in lung inflammation and inflammatory damage in a dose-dependent manner [21]. It is possible that $\mathrm{H}_{2} \mathrm{~S}$ affects the ALI/ARDS pathogenesis through mitochondria. Thus, our study aims to find the possible roles of $\mathrm{H}_{2} \mathrm{~S}$ in LPS-induced ALI in rats and its regulatory effects on the lung mitochondria with different doses.

\section{Methods}

This study was approved by the Institutional Experimental Animal Care and Ethics Committee of Hebei Medical University (Shijiazhuang, China). All animal care and experimental protocols complied with the Animal Management Rules of the Ministry of Health of the People's Republic of China (documentation number 19890503) and the Guide for the Care and Use of Laboratory Animals of Hebei Medical University (Shijiazhuang, China). The study was performed in the Animal Laboratory of our Department of Pharmacology.

\section{Animals}

This study included 40 male Sprague-Dawley rats, weighing 250-280 g, which were obtained from the Experimental Animal Centre, Hebei Medical University (Shijiazhuang, China) and kept in our Laboratory Animal Husbandry Facility until the experiments. Rats were acclimated for 1 week before experiments, with unrestricted access to deionized water and standard rat chow with no other restrictions. Before and throughout the study, rats were kept at room temperature $\left(22^{\circ} \mathrm{C}\right)$ at $30 \%-70 \%$ humidity on a $12 \mathrm{~h}$ day/night cycle with the lights turned on at 7:00 AM. No deaths occurred before intervention. All procedures were performed in accordance with the Guidelines of Animal Experiments from the Committee of Medical Ethics, National Health Department of China.

\section{Materials}

The $\mathrm{H}_{2} \mathrm{~S}$ donor NaHS was obtained from Sigma-Aldrich (St Louis, MO, USA). E.coli LPS (serotype 0127:B8) was obtained from the Sigma-Aldrich Company, Ltd. (Poole, Dorset, U.K.). SOD, MDA, GPx, and ATPase detection kits were obtained from the Nanjing Jiancheng Bioengineering Institute (Nanjing, Jiangsu, China). The primary rabbit multiclonal antibodies were obtained from Abcam Company, Ltd. (Cambridge, U.K.). All stock solutions were prepared in nonpyrogenic saline $(0.9 \% \mathrm{NaCl})$ from Shijiazhuang No.4 Pharmaceutical Company, Ltd. (Shijiazhuang, Hebei, China). 


\section{Experimental protocol}

Rats were randomly divided into five groups $(\mathrm{n}=8$ in each): control, LPS injury, LPS + low-dose NaHS $\left(0.78 \mathrm{mg} \cdot \mathrm{kg}^{-1}\right)$, LPS + middle-dose NaHS $\left(1.56 \mathrm{mg} \cdot \mathrm{kg}^{-1}\right)$, and LPS + high-dose NaHS $\left(3.12 \mathrm{mg} \cdot \mathrm{kg}^{-1}\right)$. Rats were anaesthetized with an injection of $100 \mathrm{~g} / \mathrm{L}$ chloral hydrate $\left(3 \mathrm{ml} \bullet \mathrm{kg}^{-1}\right)$. Anesthesia depth was evaluated every $2-3 \mathrm{~min}$ throughout the study. No supplemental oxygen, fluids, or mechanical ventilation were involved during the anesthesia period. The same dose was administered to the different groups. After adequate anesthesia, rats were fixed in the dorsal position on a surgical table.

1. Control group: Rats were treated with an equal volume of saline without causing endotoxemia.

2. LPS injury group: Rats were treated with E.coli LPS (5 $\mathrm{mg} \cdot \mathrm{kg}^{-1}$ via sublingual vein injections, serotype 0127:B8) lasting over $10 \mathrm{~min}$ and also treated with an equal volume of saline via intraperitoneal injections $3 \mathrm{~h}$ after LPS induction.

3. LPS + low-dose NaHS group: Rats were administered $\mathrm{NaHS}\left(0.78 \mathrm{mg} \cdot \mathrm{kg}^{-1}\right)$ via intraperitoneal injection $3 \mathrm{~h}$ after LPS induction.

4. LPS + middle-dose NaHS group: Rats were administered NaHS $\left(1.56 \mathrm{mg} \bullet \mathrm{kg}^{-1}\right)$ via intraperitoneal injection $3 \mathrm{~h}$ after LPS induction.

5. LPS + high-dose NaHS group: Rats were administered NaHS $\left(3.12 \mathrm{mg} \cdot \mathrm{kg}^{-1}\right)$ via intraperitoneal injection $3 \mathrm{~h}$ after LPS induction.

All rats were killed $3 \mathrm{~h}$ after NaHS or saline administration.

\section{Lung histology and IQA}

The middle lobe of the right lung was fixed in 10\% (wt/vol) formalin and routinely processed in paraffin sections for hematoxylin and eosin staining. Ten fields from each slice were visualized by microscopy $(\times 200)$. The average values were taken as a modified semi-quantitative histological index of quantitative assessment (IQA) of lung injury using the following criteria: 0 , no alveolitis; +1 , thickening of the alveolar septum by a mononuclear cell infiltrate, with involvement limited to focal, pleural-based lesions occupying less than $25 \%$ of the lung and with good alveolar architecture preservation; +2 , more extensive fibrosis involving 26\%-50\% of the lung and fibrotic regions, mostly extending inward from the pleura and still focal; +3 , widespread fibrosis involving $51 \%-70 \%$ of the lung; and +4 , widespread fibrosis involving more than $70 \%$ of the lung $[22,23]$.

\section{Determination of wet-to-dry lung weight ratio}

The lung wet-to-dry (W/D) weight ratio was used as an index of lung water accumulation. To measure the total amount of lung water, we measured the right lung upper lobe weight immediately after its excision (wet weight). The lung tissue was then dried in an oven at $60^{\circ} \mathrm{C}$ for $48 \mathrm{~h}$ and re-weighed as dry weight. The W/D weight ratio was calculated by dividing the wet by the dry weight, as described previously.

\section{Cytokine measurements}

Blood was drawn from the left carotid artery, and serum samples were frozen and stored at $-80^{\circ} \mathrm{C}$. Serum samples were tested using ELISA kits for interleukin-1 $\beta$ (IL-1 $\beta$ ) (R\&D Systems GmbH, Wiesbaden, Germany) according to the manufacturers' instructions.

\section{Observation of ultrastructural changes to mitochondria in lung cells}

After rats were killed, the lung tissue $\left(1 \mathrm{~mm}^{3}\right)$ was resected and fixed in $4 \%$ glutaraldehyde in $0.1 \mathrm{~mol} \cdot \mathrm{L}^{-1}$ phosphate buffer $(\mathrm{pH} 7.4)$ at $4^{\circ} \mathrm{C}$. Tissues were washed three times in dimethyl arsenate buffer and then post-fixed with $1 \%$ osmium tetroxide for $1 \mathrm{~h}$, followed by another three washes in dimethyl arsenate buffer and dehydration by passage through graded ethylene alcohol concentrations. After sequential propylene oxide treatments, ultrathin sections were cut using a Leica (Wetzlar, Germany) UCT Ultra Microtome, stained with 1\% uranyl acetate and lead citrate, and observed under a transmission electron microscope.

\section{Isolated mitochondria preparation}

Mitochondria were isolated as previously described. Briefly, after rats were killed, the lung tissues were removed rapidly into ice-cold isolation medium $\left(0.025 \mathrm{~mol} \cdot \mathrm{L}^{-1}\right.$ sucrose, $0.075 \mathrm{~mol} \cdot \mathrm{L}^{-1}$ mannitol, $0.001 \mathrm{~mol} \cdot \mathrm{L}^{-1}$ EDTA, and $\mathrm{pH}$ 7.4). The tissues were finely homogenized using a homogenizer in a glass pestle. The homogenate was centrifuged at $600 \mathrm{~g}$ for $7 \mathrm{~min}$ at $2^{\circ} \mathrm{C}$. The supernatant was collected and then centrifuged again at $1600 \mathrm{~g}$ for $5 \mathrm{~min}$ at $2^{\circ} \mathrm{C}$. The crude mitochondrial pellet was resuspended in a final volume of $5 \mathrm{~mL}$ of $3 \%$ Ficoll medium $\left(0.12 \mathrm{~mol} \cdot \mathrm{L}^{-1}\right.$ mannitol, 0.03 $\mathrm{mol} \cdot \mathrm{L}^{-1}$ sucrose, $0.025 \mathrm{~mol} \cdot \mathrm{L}^{-1} \mathrm{~K}^{+}$-EDTA, $\left.\mathrm{pH} 7.4\right)$. This suspension was carefully layered onto $10 \mathrm{~mL}$ of $6 \%$ Ficoll medium $\left(0.24 \mathrm{~mol} \cdot \mathrm{L}^{-1}\right.$ mannitol, $0.06 \mathrm{~mol} \cdot \mathrm{L}^{-1}$ sucrose, $0.05 \mathrm{~mol} \cdot \mathrm{L}^{-1} \mathrm{~K}^{+}$-EDTA, $\mathrm{pH}$ 7.4) and centrifuged at 12 $500 \mathrm{~g}$ for $10 \mathrm{~min}$ at $2^{\circ} \mathrm{C}$. The supernatant was decanted and the slight fluffy layer removed from the pellet. The mitochondrial pellet was resuspended in isolation medium and centrifuged again at $12500 \mathrm{~g}$ for $10 \mathrm{~min}$ at $2^{\circ} \mathrm{C}$. The prepared mitochondria were diluted in isolation medium prior to use.

\section{Determining lung mitochondrial MDA content, SOD, and GPx and ATPase activity}

SOD levels, GPx activity, ATPase activity, and MDA content in the lung mitochondria were measured with an enzyme-linked immunosorbent assay (ELISA) 
and commercially available kits (Nanjing Jiancheng Bioengineering, Nanjing, Jiangsu, China) according to the manufacturer's instructions. The kit was maintained at room temperature $\left(20-25^{\circ} \mathrm{C}\right)$ prior to testing and the washing buffer prepared $15 \mathrm{~min}$ before use. The $100 \mu \mathrm{l}$ of SOD standards and $100 \mu \mathrm{l}$ of samples were added to the corresponding plate wells and shaken gently for $30 \mathrm{~s}$ before sealing. After $1 \mathrm{~h}$ of incubation at $37^{\circ} \mathrm{C}$, the liquid was removed, and the plates were washed with washing buffer $(350 \mu \mathrm{l} /$ well $)$ and soaked for a few minutes. Plates were blotted dry by tapping upside down on filter paper. We repeated the washing five times. The $100 \mu \mathrm{l}$ of biotin was added to each plate well and left for $1 \mathrm{~h}$ at $37^{\circ} \mathrm{C}$. After five additional washing steps, $100 \mu \mathrm{l}$ of horseradish peroxides (HRP) was added to the wells and left for $30 \mathrm{~min}$ at $37^{\circ} \mathrm{C}$. The plates were again washed five times, $100 \mu \mathrm{l}$ of tetramethylbenzidine (TMB) substrate was added to each well, and plates were shaken gently for $10 \mathrm{~s}$. The mixture was incubated in the dark for $(15 \pm 10) \mathrm{min}$ at $37^{\circ} \mathrm{C}$. Optical density (OD) at $450 \mathrm{~nm}$ was measured by ELISA reader after adding $100 \mu \mathrm{l}$ of stop solution to each well and shaking gently for $30 \mathrm{~s}$. The standard curve of OD value versus concentration was plotted. The sample data were plotted on the standard curve and the sample SOD concentration obtained. Other samples were obtained in a similar way.

\section{Determining lung mitochondria swelling}

Freshly prepared mitochondria without repeated freezethaw were kept at $4^{\circ} \mathrm{C}$ prior to the reaction. The mitochondria were removed rapidly into the medium $\left(0.025 \mathrm{~mol} \cdot \mathrm{L}^{-1}\right.$ sucrose, $0.0005 \mathrm{~mol} \cdot \mathrm{L}^{-1} \mathrm{KH}_{2} \mathrm{PO}_{4}, 0.001 \mathrm{~mol} \cdot \mathrm{L}^{-1}$ Sodium succinate, and $\mathrm{pH}$ 7.2). Mitochondrial protein content was adjusted to $0.5 \mathrm{mg} \cdot \mathrm{ml}^{-1}$. Mitochondrial swelling was determined in all groups by measuring the change in the absorbance of the lung mitochondrial suspension at $540 \mathrm{~nm}$ (Optical density 540,OD540) on a spectrophotometer at 722 . The reaction conditions were set at $25^{\circ} \mathrm{C}$.

\section{Determining of the lung mitochondria activity}

Freshly prepared mitochondria suspension $(100 \mu \mathrm{L})$ without repeated freeze-thaw was removed rapidly into the microtiter plate's microporous. We added MTT (5 $\left.\mathrm{g} \cdot \mathrm{L}^{-1,} 40 \mu \mathrm{L}\right)$ at $30^{\circ} \mathrm{C}$ and incubated it for $30 \mathrm{~min}$, then added isopropanol $(100 \mu \mathrm{L})$ for $20 \mathrm{~min}$. Colorimetric analysis was set at $570 \mathrm{~nm}$. OD570 value size indicated mitochondrial activity.

\section{Determining lung mitochondrial and cytosol Cyt-c protein expression}

After rats were killed, lung tissues were removed rapidly into ice-cold isolation medium $(0.025 \mathrm{~mol} / \mathrm{L}$ sucrose, $0.075 \mathrm{~mol} / \mathrm{L}$ mannitol, $0.001 \mathrm{~mol} / \mathrm{L}$ EDTA, and $\mathrm{pH}$ 7.4). The tissues were finely homogenized using a homogenizer in a glass pestle. The homogenate was centrifuged at $600 \mathrm{~g}$ for $7 \mathrm{~min}$ at $2^{\circ} \mathrm{C}$. The supernatant was collected and then centrifuged again at $1600 \mathrm{~g}$ for $5 \mathrm{~min}$ at $2^{\circ} \mathrm{C}$. The crude mitochondrial pellet was resuspended in a final volume of $5 \mathrm{~mL}$ of $3 \%$ Ficoll medium $(0.12 \mathrm{~mol} / \mathrm{L}$ mannitol, $0.03 \mathrm{~mol} / \mathrm{L}$ sucrose, $0.025 \mathrm{~mol} / \mathrm{L} \mathrm{K}^{+}$-EDTA, $\mathrm{pH}$ 7.4). This suspension was carefully layered onto $10 \mathrm{~mL}$ of $6 \%$ Ficoll medium $(0.24 \mathrm{~mol} / \mathrm{L}$ mannitol, $0.06 \mathrm{~mol} / \mathrm{L}$ sucrose, $0.05 \mathrm{~mol} / \mathrm{L} \mathrm{K} \mathrm{K}^{+}$-EDTA, $\mathrm{pH}$ 7.4) and centrifuged at $12500 \mathrm{~g}$ for $10 \mathrm{~min}$ at $2^{\circ} \mathrm{C}$. The supernatant was decanted and the slight fluffy layer removed from the pellet. The mitochondrial pellet was resuspended in isolation medium and centrifuged again at $12500 \mathrm{~g}$ for $10 \mathrm{~min}$ at $2^{\circ} \mathrm{C}$. The mitochondria and cytosol supernatant were used for cytochrome c (Cyt-c) analysis using Western blotting assay.

Protein extractions in each group were subjected to $10 \%$ sodium dodecyl sulfate-polyacrylamide gel electrophoresis (SDS-PAGE) and transferred onto a polyvinylidene difluoride (PVDF) membrane. The membranes were treated with blocking solution (5\% skim milk in TBST) and incubated overnight at $4^{\circ} \mathrm{C}$ with the primary rabbit multiclonal antibodies, respectively (Cyt-c, 1:1000; abcam, U.K.). Immunoblots were developed with horseradish peroxidaseconjugated secondary antibodies, visualized using an enhanced chemiluminescence reagent (Millipore, Billerica, MA, USA), and quantified by densitometry using a ChemiDoc XRS (Bio-Rad, Berkeley, California, USA). The band density was normalized to $\beta$-actin. The proteins' increase or decrease percentage was estimated by comparison to vehicle control (100\%).

\section{Statistical analysis}

Based on the assumed differences and variability in the data marking a biologic effect of treatment in each group, numbers of animals per group were estimated before the study. All data are presented as the mean \pm SD. Differences between groups were assessed by one-way ANOVA followed by Tukey's post hoc multiple comparison test using SPSS, version 13.0 (SPSS, Armonk, NY, USA). IQA scores were achieved using the rank-sum test. Two-sided $\mathrm{P}$ values of less than 0.05 were considered statistically significant.

\section{Results}

\section{Histological lung tissue changes in each group}

We found that the control group morphology was normal with light microscopy, and there was no alveolar edema fluid in the alveolar space. The alveolar wall was intact with no evidence of inflammatory cell infiltration or hemorrhage. Conversely, the LPS injury group showed diffuse edema in alveolar spaces, lung interstitium, hemorrhage, severe inflammatory cell infiltration, serous exudation in the alveolar space, and a thickened interbular 
septa like "hyaline membrane". These changes were lightly mitigated in the LPS + low-dose NaHS group, and significantly mitigated in LPS + middle-dose and high-dose NaHS groups (Figure 1).

\section{IQA scores in each group}

Significantly higher IQA scores were observed in the LPS group ( $3.12 \pm 0.02$ vs. $0.05 \pm 0.08, P<0.01)$ compared to the control group, but IQA scores were slightly lower in the LPS + low-dose NaHS group $(2.52 \pm 0.12$ vs. $3.12 \pm 0.02, P<0.01$ ) and significantly lower in the LPS + middle-dose $(2.47 \pm 0.20$ vs. $3.12 \pm 0.02, P<0.01)$ and high-dose NaHS groups ( $2.32 \pm 0.15$ vs. $3.12 \pm 0.02$, $P<0.01)$ compared to the LPS group.

\section{W/D lung weight ratios}

The W/D lung weight ratios were significantly increased in the LPS group $(5.52 \pm 0.20$ vs. $4.73 \pm 0.32, P<0.01)$ compared to the control group. Compared to the LPS group, the W/D lung weight ratios were respectively decreased in LPS + low-dose $(5.20 \pm 0.27$ vs. $5.52 \pm 0.20$, $P<0.05)$, middle-dose $(4.69 \pm 0.41$ vs. $5.52 \pm 0.20, P<0.01)$, and high-dose $(4.84 \pm 0.22$ vs. $5.52 \pm 0.20, P<0.01)$ NaHS groups.

\section{IL-1 $\beta$ content in serum}

Compared to the control group, the IL- $1 \beta$ serum content $(15.03 \pm 0.82 \mathrm{pg} / \mathrm{ml}$ vs. $10.58 \pm 1.06 \mathrm{pg} / \mathrm{ml}, P<0.01)$ was significantly increased in the LPS group. Compared to the LPS group, the IL- $1 \beta$ serum content was significantly deceased in the LPS + low-dose $(13.96 \pm 0.72 \mathrm{pg} / \mathrm{ml}$ vs.

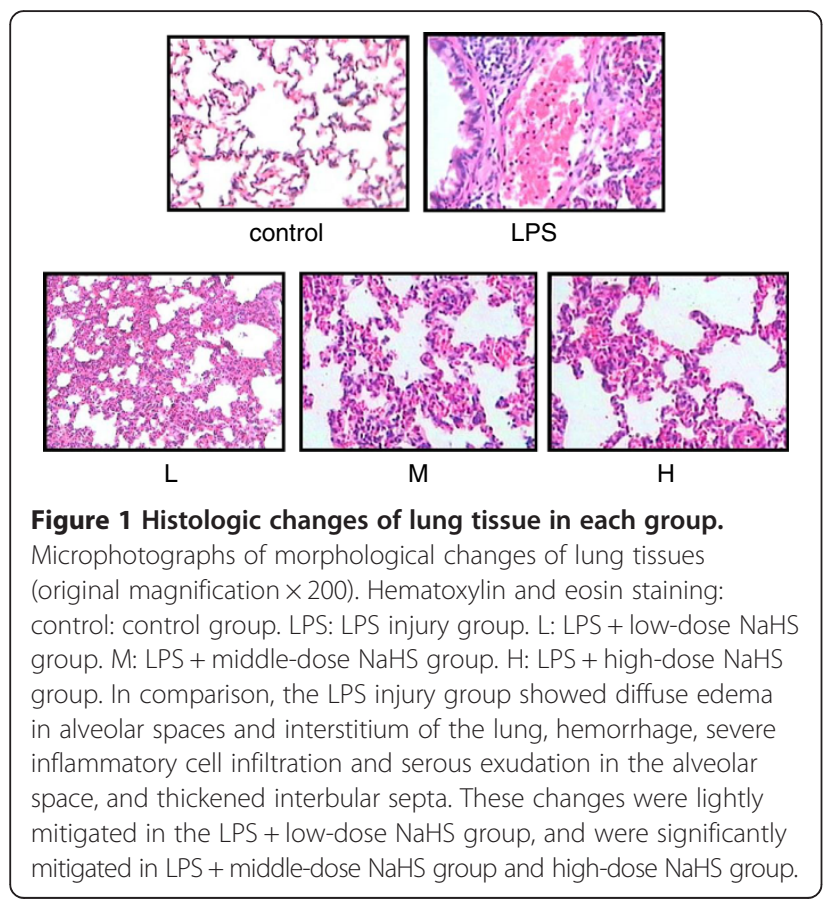

$15.03 \pm 0.82 \mathrm{pg} / \mathrm{ml}, P<0.05)$, middle-dose $(12.46 \pm 1.33$ $\mathrm{pg} / \mathrm{ml}$ vs. $15.03 \pm 0.82 \mathrm{pg} / \mathrm{ml}, P<0.01)$, and high-dose $(12.23 \pm 0.89 \mathrm{pg} / \mathrm{ml}$ vs. $15.03 \pm 0.82 \mathrm{pg} / \mathrm{ml}, P<0.01) \mathrm{NaHS}$ groups.

\section{Effects of $\mathrm{H}_{2} \mathrm{~S}$ on mitochondrial ultrastructure in lung cells}

There were significant differences between the mitochondrial ultrastructure in the control and LPS groups' lung cells. The mitochondria in lung cells from the LPS injury group were swollen with disrupted or disintegrated cristae, and the osmiophilic lamellar bodies had fused or disappeared. This mitochondrial damage was slightly mitigated in the LPS + low-dose NaHS group and significantly mitigated in the LPS + middle-dose and high-dose NaHS groups (Figure 2).

Effects of $\mathrm{H}_{2} \mathrm{~S}$ on MDA content and mitochondrial ATPase, SOD, and GSH-P activity

Compared to the control group, the MDA content was significantly increased $(P<0.01)$ and SOD, GSH-PX, and ATPase activities significantly decreased $(P<0.01)$ in lung mitochondria in the LPS group. Compared to the LPS group, the MDA content was significantly decreased and SOD, GSH-PX, and ATPase activities significantly increased in the three LPS + NaHS groups $(P<0.05$ or $P<0.01$ ) (Figures 3, 4, 5 and 6).

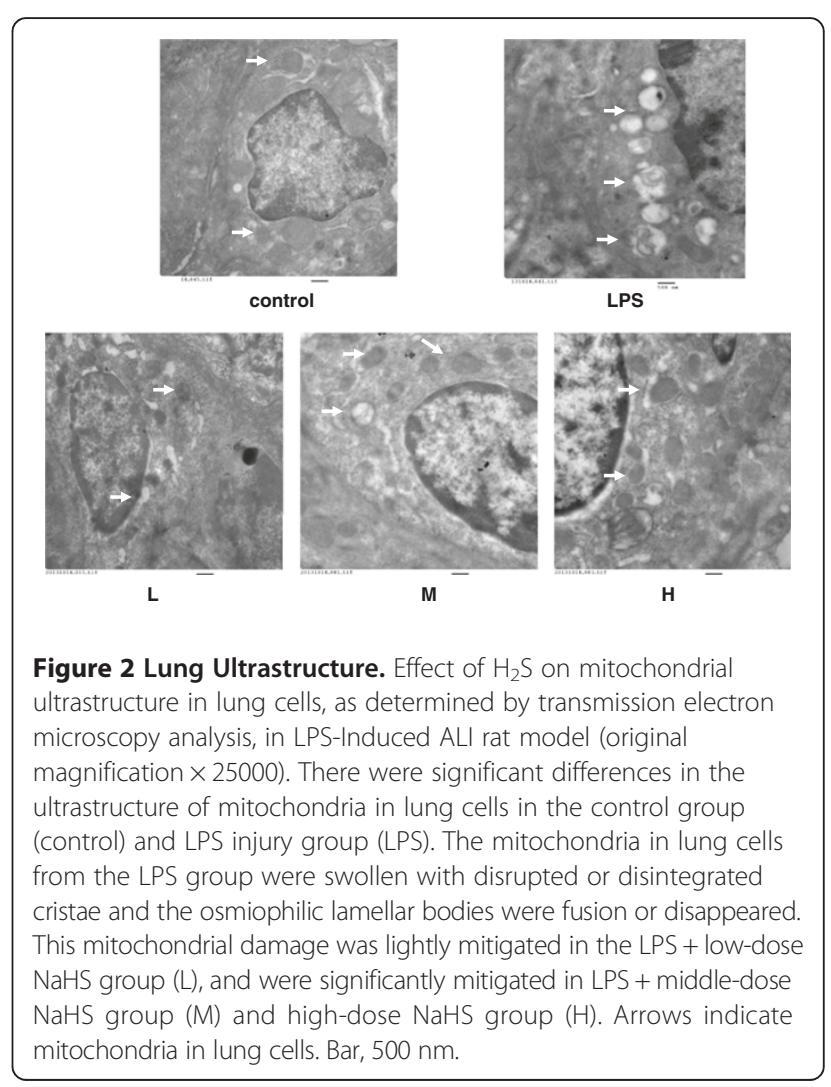




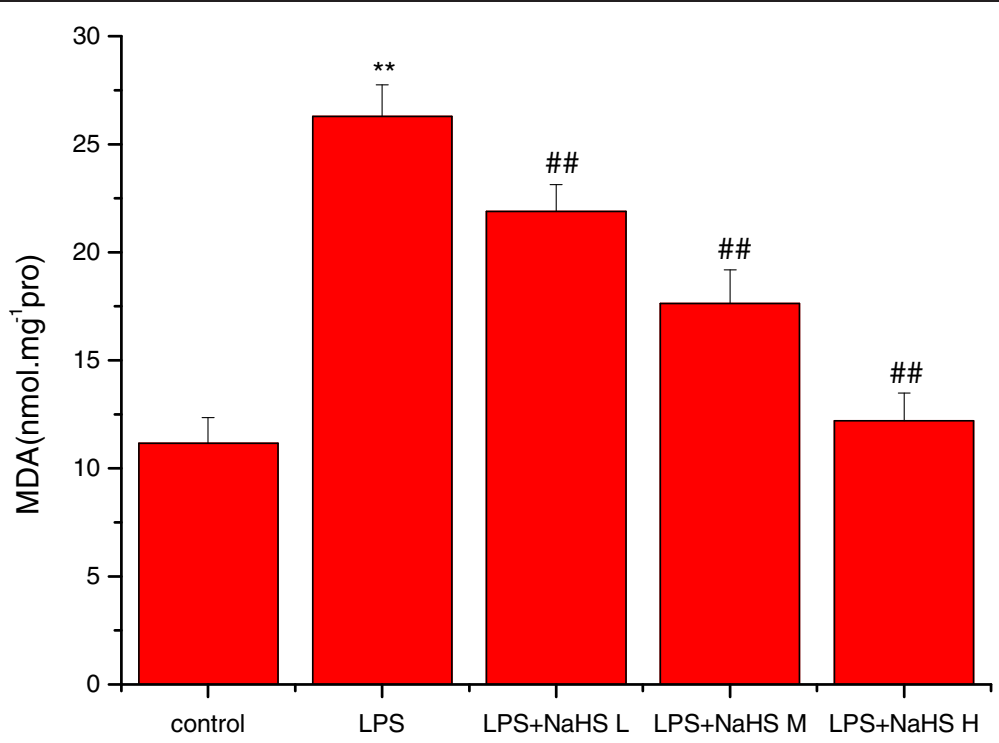

Figure 3 Effect of $\mathrm{H}_{2} \mathrm{~S}$ on the content of MDA in lung mitochondria in rats. Compared with control group, the content of MDA was significantly increased $(P<0.01)$ in lung mitochondria in LPS injury group. Compared with LPS injury group, the content of MDA was significantly decreased in LPS + low, middle and high dose NaHS groups $(P<0.01)$. ${ }^{* *} P<0.01$ vs control; \#\#P<0.01 vs LPS. control: control group. LPS: LPS injury group.LPS+ NaHS L: LPS + low-dose NaHS group.LPS+ NaHS M: LPS + middle-dose NaHS group.LPS+ NaHS H: LPS + high-dose NaHS group.

Effects of $\mathrm{H}_{2} \mathrm{~S}$ on lung mitochondria swelling and activity Mitochondrial permeability transition (MPT) is an important index to indicate the integrity and function of mitochondria.The principle of measurement for MPT is to observe the change of absorbance at $540 \mathrm{~nm}$ (OD540), due to the swelling of mitochondria by the disturbance of permeability transition of mitochondrial membrane when MPT increase. Decreased absorbance (the OD540 value) indicates mitochondrial swelling $[24,25]$. Mitochondrial activity was assessed by the MTT colorimetric assay using OD570. The swelling of the mitochondria was significantly increased (the OD540 value was significantly decreased) and the activity of the mitochondria was significantly decreased in the LPS compared with the control group $(P<0.01)$. In the three LPS + NaHS groups, the swelling of the

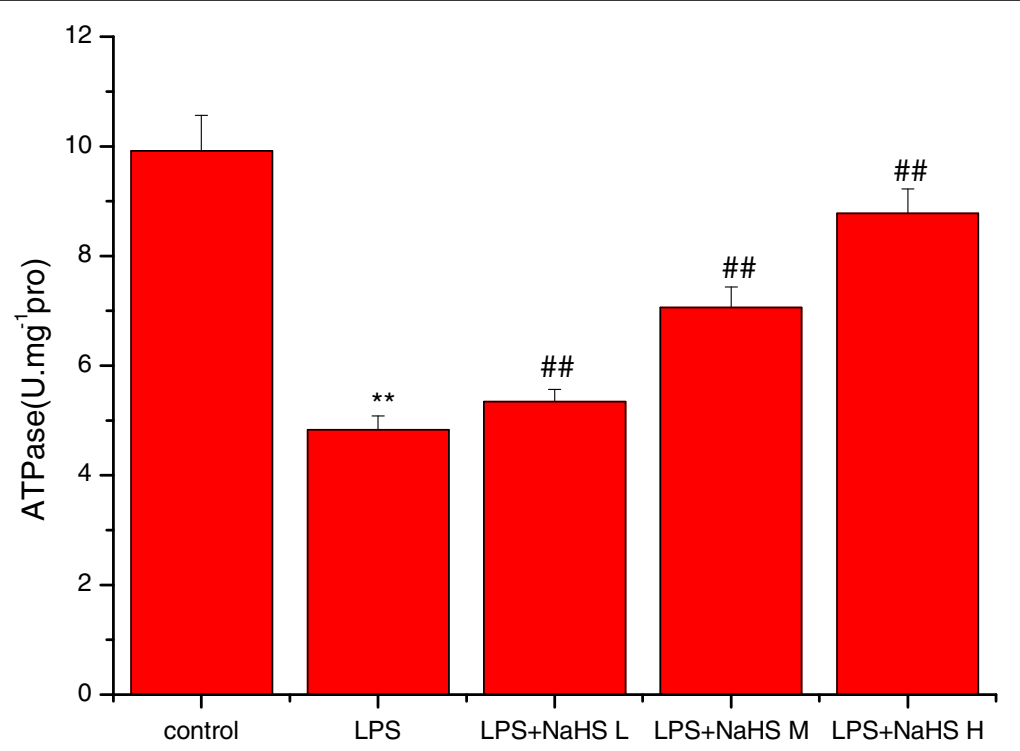

Figure 4 Effect of $\mathrm{H}_{2} \mathrm{~S}$ on the activity of ATPase in lung mitochondria in rats. Compared with control group, the activities of ATPase were significantly decreased $(P<0.01)$ in lung mitochondria in LPS injury group. Compared with LPS injury group, the activities of ATPase were significantly increased in LPS + low, middle and high dose NaHS groups $(P<0.01)$. ${ }^{*} P<0.01$ vs control; \#\#P<0.01 vs LPS.control: control group. LPS: LPS injury group.LPS+ NaHS L: LPS + low-dose NaHS group.LPS+ NaHS M: LPS + middle-dose NaHS group.LPS+ NaHS H: LPS + high-dose NaHS group. 


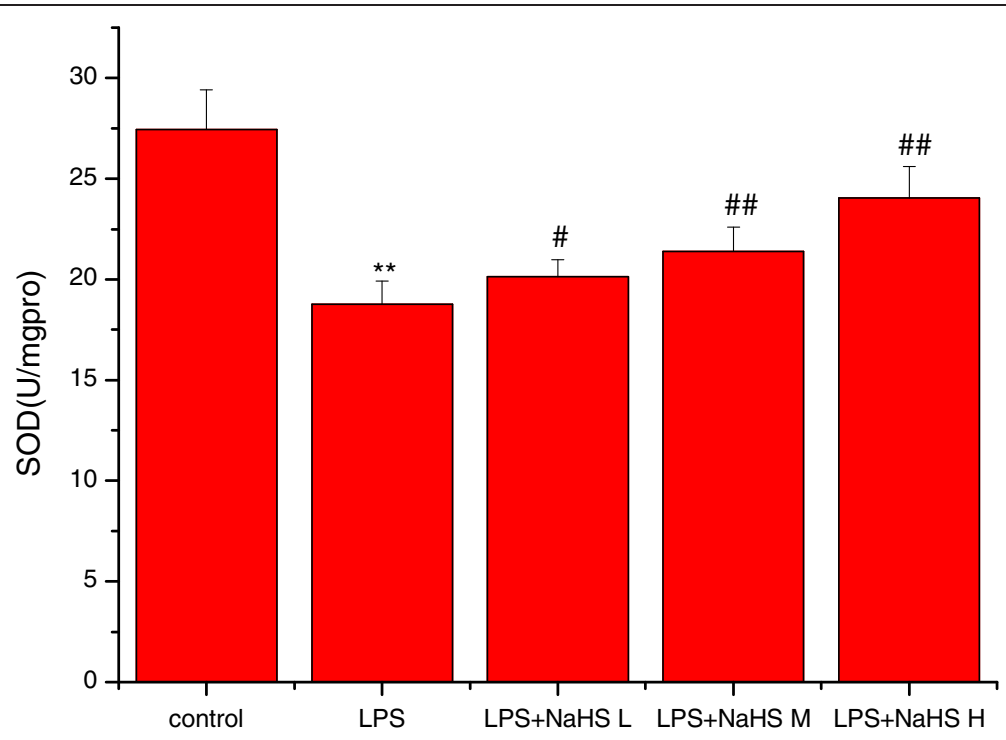

Figure 5 Effect of $\mathrm{H}_{2} \mathrm{~S}$ on the activity of SOD in lung mitochondria in rats. Compared with control group, the activities of SOD were significantly decreased $(P<0.01)$ in lung mitochondria in LPS injury group. Compared with LPS injury group, the activities of SOD were significantly increased in LPS + low, middle and high dose NaHS groups $(P<0.05$ or $P<0.01)$. ${ }^{* *} P<0.01$ vs control; \#P<0.05, \#\#P<0.01 vs LPS. control: control group. LPS: LPS injury group.LPS+ NaHS L: LPS + low-dose NaHS group.LPS+ NaHS M: LPS + middle-dose NaHS group.LPS+ NaHS H: LPS + high-dose NaHS group.

mitochondria was markedly decreased (the OD540 value was significantly increased) and the activity of the mitochondria was markedly increased compared with the LPS group $(\mathrm{P}<0.05$ or $\mathrm{P}<0.01)$ (Figures 7 and 8).

\section{Effects of $\mathrm{H}_{2} \mathrm{~S}$ on lung mitochondrial and cytosol Cyt-c} protein expression

The band intensity showed the Cyt-c protein expression. The Cyt-c protein expression in the lung mitochondria was significantly decreased in the LPS injury group

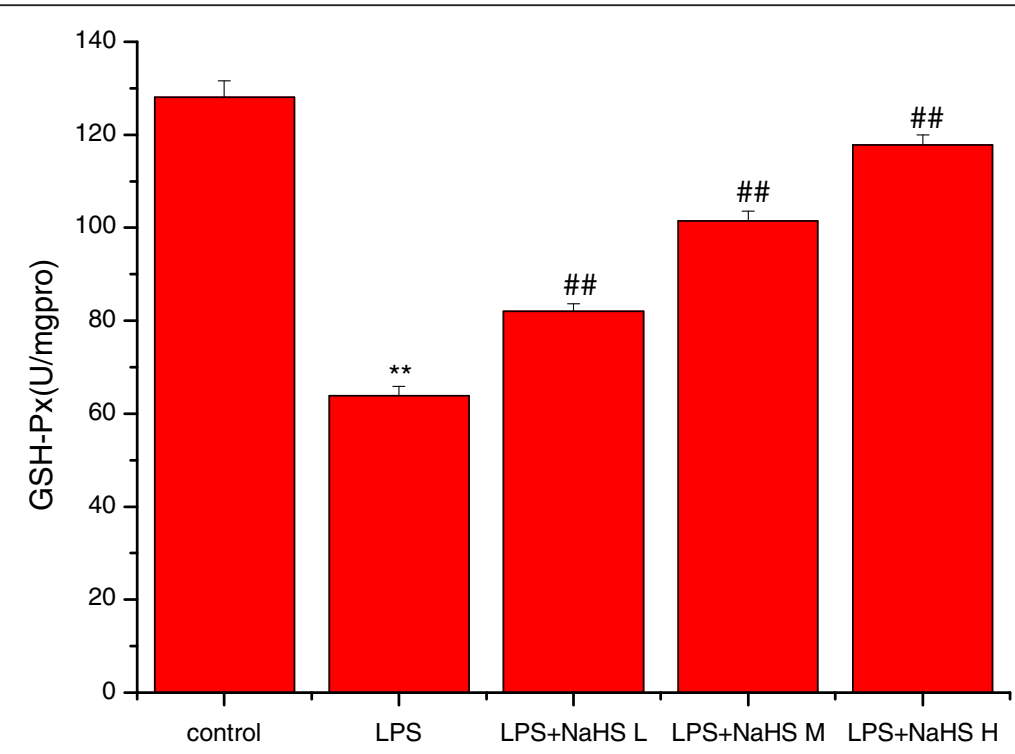

Figure 6 Effect of $\mathrm{H}_{2} \mathrm{~S}$ on the activity of GSH-P in lung mitochondria in rats. Compared with control group, the activities of GSH-PX were significantly decreased $(P<0.01)$ in lung mitochondria in LPS injury group. Compared with LPS injury group, the activities of GSH-Px were significantly increased in LPS + low, middle and high dose NaHS groups $(P<0.01)$. ${ }^{* *} P<0.01$ vs control; \#\#P<0.01 vs LPS.control: control group. LPS: LPS injury group.LPS+ NaHS L: LPS + low-dose NaHS group.LPS+ NaHS M: LPS + middle-dose NaHS group.LPS+ NaHS H: LPS + high-dose NaHS group. 


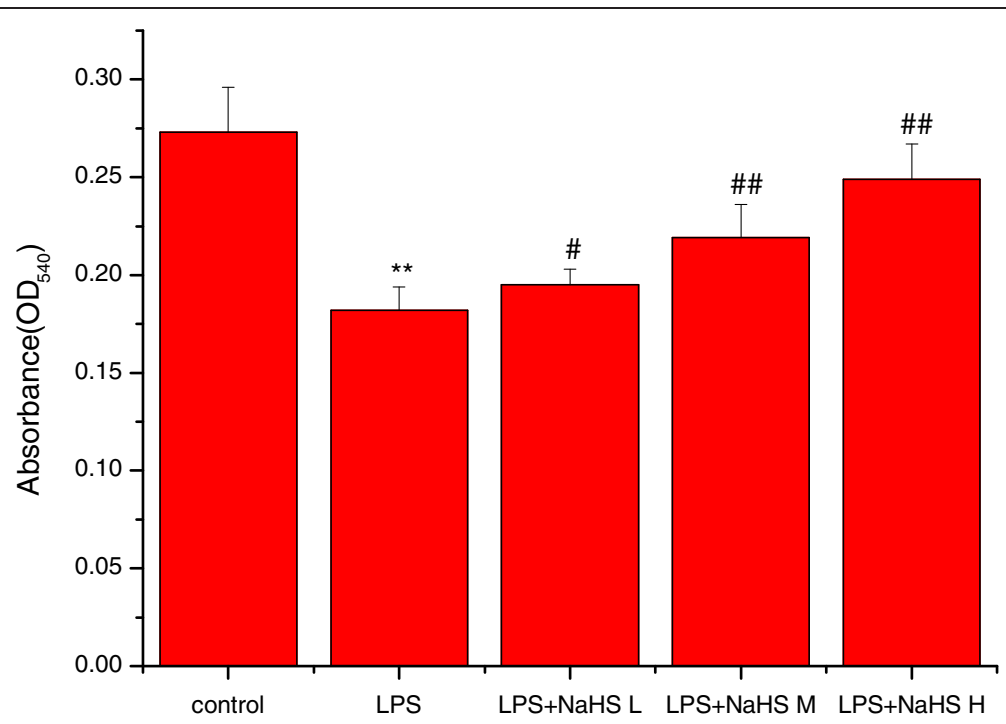

Figure 7 Effect of $\mathrm{H}_{2} \mathrm{~S}$ on the swelling of lung mitochondria in rats. The swelling extent of the mitochondria was significantly increased (the OD540 value was significantly decreased) in the LPS injury group compared with the control group ( $P<0.01)$. In LPS + low-dose NaHS group, LPS + middle-dose NaHS group and LPS + high-dose NaHS group, the swelling of the mitochondria was markedly decreased (the OD540 value was significantly increased) with the LPS injury group $(P<0.05$ or $P<0.01)$. ${ }^{*} P<0.01$ vs control; $\# P<0.05$, \#\#P<0.01 vs LPS.control: control group. LPS: LPS injury group.LPS+ NaHS L: LPS + low-dose NaHS group.LPS+ NaHS M: LPS + middle-dose NaHS group.LPS+ NaHS H: LPS + high-dose NaHS group.

compared to the control group $(P<0.01)$. In the three LPS + NaHS groups, Cyt-c protein expression in the lung mitochondria was markedly increased compared to the LPS injury group $(P<0.01)$. The cytosol Cyt-c protein expression was significantly increased in the LPS group compared to the control group $(P<0.01)$. In the three LPS + NaHS groups, cytosol Cyt-c protein expression was markedly decreased compared to the LPS injury group $(P<0.01)$ (Table 1 , Figure 9$)$.

\section{Discussion}

ALI/ARDS is a common clinical illness. The current ALI mortality rate is as high as $35 \%-40 \%$ and reaches to $50 \%$ in ARDS [26,27]. ALI's pathogenesis is complex, and

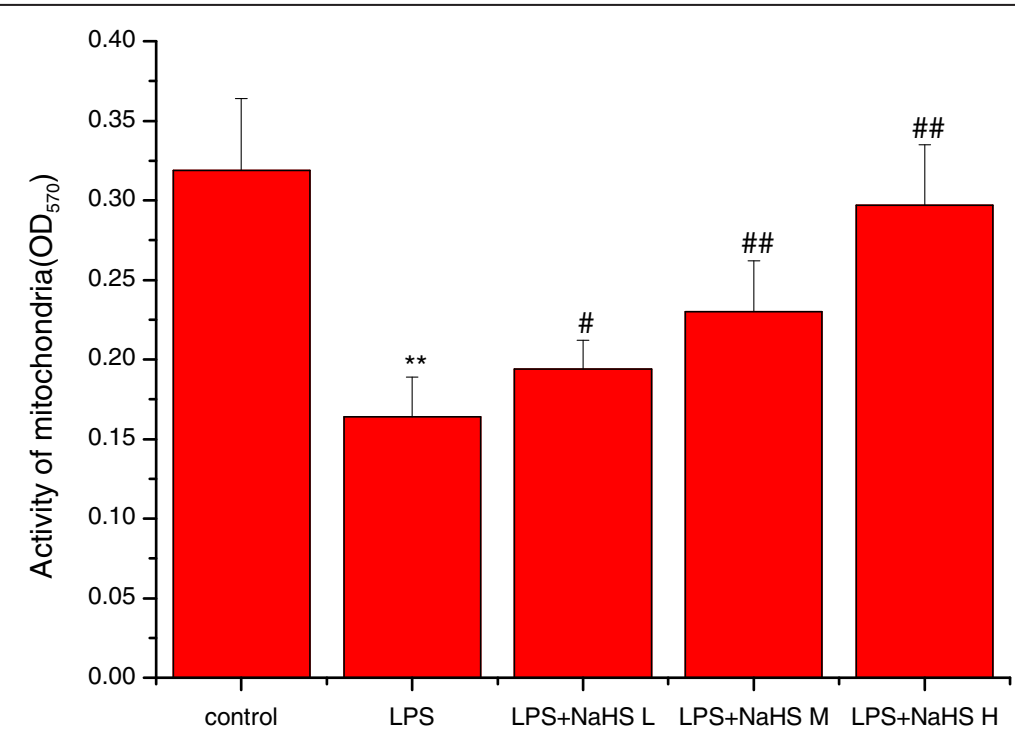

Figure 8 Effect of $\mathrm{H}_{2} \mathrm{~S}$ on the activity of lung mitochondia in rats. The activity of the mitochondria was significantly decreased in the LPS injury group compared with the control group $(P<0.01)$. In LPS + low-dose NaHS group,LPS + middle-dose NaHS group and LPS + high-dose NaHS group, the activity of the mitochondria was markedly increased compared with the LPS injury group $(P<0.05$ or $P<0.01)$. ${ }^{* *} P<0.01$ vs control; \#P<0.05, \#\#P<0.01 vs LPS.control: control group. LPS: LPS injury group.LPS+ NaHS L: LPS + low-dose NaHS group.LPS+ NaHS M: LPS + middle-dose NaHS group.LPS+ NaHS H: LPS + high-dose NaHS group. 
Table 1 Effects of NaHS on lung mitochondrial and cytosol Cyt-c protein expression in acute lung injury rats $(\bar{x} \pm \mathbf{s}, \mathbf{n}=\mathbf{8})$

\begin{tabular}{|c|c|c|c|}
\hline Group & Cyt-c (mitochondria) (Grayscale scan value) & Cyt-c (cytosol) (Grayscale scan value) & $\beta$-actin (Grayscale scan value) \\
\hline control group & 178.51 & 96.992 & 171.47 \\
\hline LPS injury group & $41.177^{* *}$ & $293.45 * *$ & 183.66 \\
\hline L & $62.846^{\#}$ & $226.91^{\#}$ & 182.52 \\
\hline M & $88.344^{\# \#}$ & $203.38^{\# \#}$ & 196.02 \\
\hline H & $115.27^{\# \#}$ & $111.84^{\# \#}$ & 169.28 \\
\hline
\end{tabular}

${ }^{* *} P<0.01$, compared to the control group; ${ }^{\#} P<0.05,{ }^{\# \#} P<0.01$, compared to the LPS injury group; L:LPS + low-dose NaHS group; M:LPS + middle-dose NaHS group; $\mathrm{H}$ : LPS + high-dose NaHS group.

The lung mitochondrial Cyt-c protein expression was significantly decreased in the LPS injury group compared to the control group ( $P<0.01)$. In three LPS + NaHS groups, lung mitochondrial Cyt-c protein expression was markedly increased compared to the LPS injury group $(P<0.05$ or $P<0.01)$. The cytosol Cyt-c protein expression was significantly increased in the LPS injury group compared to the control group $(P<0.01)$. In three LPS $+\mathrm{NaHS}$ groups, cytosol Cyt-c protein expression was markedly decreased compared to the LPS injury group $(P<0.05$ or $P<0.01)$.

there is still much controversy to be had before we reach a definitive conclusion [28]. Increased oxidative stress has been implicated in its pathogenesis $[29,30]$. The lung-protective, low-tidal-volume ventilation strategy increases survival rate by limiting alveolar damage and consequent biotrauma [31], but ideal targeted drug therapies have been shown to be curative. Therefore, alternative strategies are urgently needed to improve care. The LPS-induced ALI rat model is a classic animal model [5]. In our ALI rat model, LPS-treated rats showed higher IQA scores than control rats, which is in compliance with ALI's clinical manifestations [32]. Thus, injecting LPS via the sublingual vein successfully induced ALI in rats, as reported previously [5].

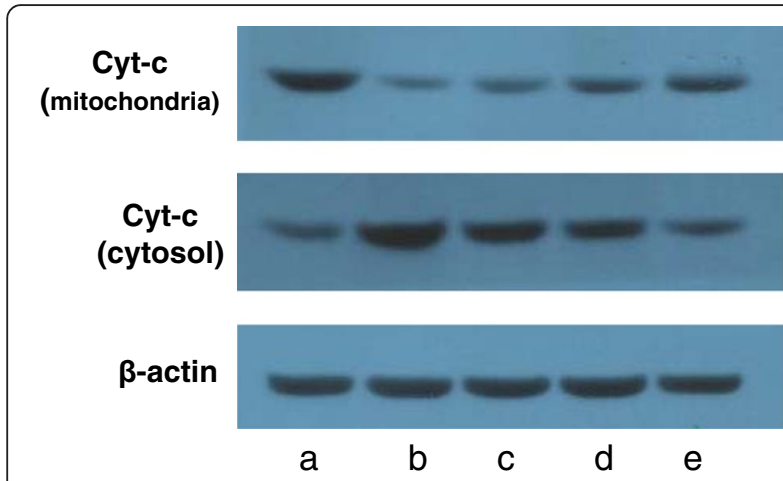

Figure 9 The expression of lung mitochondrial Cyt-c and cytosol Cyt-c were detected by Western blotting method. The Cyt-c protein expression of the lung mitochondria was significantly decreased in the LPS injury group compared with the control group $(P<0.01)$. In the LPS + low-dose, middle-dose and high-dose NaHS group, Cyt-c protein expression of the lung mitochondria was markedly increased compared with the LPS injury group $(P<0.05$ or $P<0.01)$. The Cyt-c protein expression of cytosol was significantly increased in the LPS compared with the control group $(P<0.01)$. In the LPS + low-dose, middle-dose and high-dose NaHS group, Cyt-c protein expression of cytosol was markedly decreased compared with the LPS injury group $(P<0.05$ or $P<0.01)$. a: control group. b: LPS injury group. c: LPS+ low-dose NaHS group. d: LPS + middle-dose NaHS group. e: LPS + high-dose NaHS group.
In recent years, some researchers found that mitochondrial dysfunction plays an important role in the course of ALI [33]. Mitochondria utilize approximately $98 \%$ of total body oxygen consumption [34,35]. This would maintain tissue oxygen levels by decreasing demand and protect against cell death [36]. Studies have shown that mitochondrial dysfunction is the key factor to cell damage [37]. In sepsis, mitochondrial dysfunction in vital organs can make cell organisms lack energy, causing multiple organ failure [38]. The lung is a special organ relatively susceptible to injury [39]. The mitochondrion is a complex and sensitive organelle. ALI can lead to abnormal mitochondrial structure and function and tends to cause abnormal mitochondria organelles or other changes in the entire cell, thereby increasing the degree of ALI. Meanwhile, mitochondrial dysfunction is also prone to result in ALI/ARDS [40].

Including the case of LPS-induced lung injury, lung tissue can produce large radical $\mathrm{NO}, \mathrm{O}^{-2}$, and $\mathrm{ONOO}^{-}$. The mitochondrial film, which is rich in unsaturated fatty acids, is a major free radical attack target. These lead to mitochondrial membrane swelling expansion, lipid oxidation increase, and decrease membrane fluidity. Mitochondrial ATP enzyme activity and mitochondrial ATP production were also decreased because of these. Mitochondria have intrinsic defense mechanisms to protect against ROS-induced damage through its large array of antioxidants (e.g., superoxide dismutase, glutathione, thioredoxin) [41]. Under physiological conditions, mitochondrial SOD and GSH-Px content are rich. They timely remove oxygen free radicals. In ALI, cells produce large amounts of oxygen free radicals, resulting in mitochondrial SOD and GSH-Px activities. ATP production decreases while consumption and MDA content increase. Ultimately, these lead to mitochondrial structure destruction [42].

$\mathrm{H}_{2} \mathrm{~S}$ is a gaseous mediator that may be a promising therapy for preserving organ function and life during suspended animation in vivo models [43]. Major targets clearly include the inhibition of mitochondrial cytochrome 
c oxidase and activation of endothelial cell $\mathrm{K}^{+}$-ATPase channels, but the downstream effects of both pathways are highly context-dependent. Potential utility of $\mathrm{H}_{2} \mathrm{~S}$ in sepsis has been demonstrated in several animal studies with improvements in organ function and survival [44-46]. Our experimental results showed that, compared to the control group, IQA scores, W/D weight ratios, IL-1 $\beta$ serum content, lung mitochondrial MDA content, and mitochondrial swelling in the LPS injury group were significantly increased, and mitochondria, ATPase, SOD, and GSH-Px activities were significantly decreased. The lung mitochondrial Cyt-c protein expression was significantly decreased, the cytosol Cyt-c protein expression was significantly increased (Cyt-c was released from mitochondria), and the lung mitochondrial ultrastructure was damaged. Compared to the LPS injury group, the NaHS treatment group's IQA scores, W/D weight ratio, IL-1 $\beta$ serum content, mitochondrial MDA content, and mitochondrial swelling were significantly decreased, and mitochondria, ATPase, SOD, and GSH-Px activities were significantly increased. The lung mitochondrial Cyt-c protein expression was significantly increased, the cytosol Cyt-c protein expression was significantly decreased (suppressed the release of Cyt-c from mitochondria), and the lung mitochondrial ultrastructure damage looked better. Meanwhile, these therapeutic roles were dose-dependent (positively correlated with the dosage). The mechanism for this could be that $\mathrm{H}_{2} \mathrm{~S}$ suppressed the mitochondrial cytochrome $\mathrm{c}$ oxidase, decreased the level of mitochondrial lipid peroxidation, and protected cell structure. However, conflicting data exists in models of cecal ligation and puncture demonstrating aggravation [47,48]. It is likely that $\mathrm{H}_{2} \mathrm{~S}$ donor purity, administration route, timing, and dosage may be accountable for the inconsistent data. Our experiment has confirmed that $\mathrm{H}_{2} \mathrm{~S}$ can reduce mitochondrial oxidative damage by free radicals, thereby reducing lung tissue damage in ALI and playing a therapeutic role in a dose-dependent manner.

\section{Limitations}

The most common experimental ALI models are caused by sepsis, including injection (local or systemic) of bacterial products like endotoxin (LPS), a commonly used organism called Escherichia coli, or lipoteichoic acid. Other models include extrusion of fecal contents from surgically manipulated gut areas [usually cecum, cecal ligation, and puncture (CLP)]. The colon ascendens stent peritonitis (CASP) causes an intraperitoneal leak of feces, leading to polymicrobial sepsis, similar to what is seen in the CLP model. Each of these models has advantages and disadvantages with dramatic differences between response timing (such as when cytokine/ chemokine levels peak in plasma). In rodents injected with LPS, plasma mediators peak in the first several hours versus in CLP, with mediator peaks developing much more slowly over the first $48 \mathrm{~h}$. In future studies, we will also use other models.

Lung tissue $\mathrm{H}_{2} \mathrm{~S}$ concentrations were not measured in this study. Comparing the lung tissue concentrations of sulfide species in future studies may help explain the differential biological effects of intravascular $\mathrm{NaHS}$ and its dose-dependency.

A recent study [49] suggested that $\mathrm{H}_{2} \mathrm{~S}$ may protect the lung against injury and drive epithelial cell migration and wound repair. This idea is supported by the increase of the activated Akt fraction (Protein kinase B). Our study does not involve the impact of $\mathrm{H}_{2} \mathrm{~S}$ on Akt pathway activation.

\section{Conclusions}

In our model, LPS resulted in lung mitochondrial structure injury and loss of function in ALI rats. Hydrogen sulfide decreased lung water, decreased the level of inflammation, reduced LPS-induced lung mitochondrial oxidative damage, and protected mitochondrial structure and function. This therapeutic role is dose-dependent. Its regulatory effect on lung mitochondria is positively correlated with the dose. Further studies are needed to reveal its potential mechanism.

\section{Key messages}

- The in vivo experimental study demonstrated that hydrogen sulfide (considered as the third gaseous transmitter) donor, NaHS, can prevent mitochondrial oxidative damage and protect mitochondrial structure and function in LPS-induced ALI rat models.

- NaHS reduced ALI-induced oxidative stress.

- NaHS reduced ALI-induced mitochondrial dysfunction.

- NaHS up-regulated the lung mitochondrial Cyt-c protein expression and down-regulated the cytosol Cyt-c protein expression (suppressed the release of Cyt-c from mitochondria) in this model.

- NaHS reduced ALI-induced mitochondrial structural damage.

- Regulatory effect of NaHS on lung mitochondria is positively correlated with the dose.

\section{Abbreviations}

ALl: Acute lung injury; ARDS: Acute respiratory distress syndrome; LPS: Lipopolysaccharide; SIRS: Systemic inflammatory response syndrome; PMN: Polymophonuclear neutrophil; NO: Nitric oxide; CO: Carbon monoxide; $\mathrm{H}_{2} \mathrm{~S}$ : Hydrogen sulfide; NaHS: Sodium hydrosulfide; ROS: Radical oxygen species; OONOO-: Peroxynitrite; IQA: Index of quantitative assessment; MDA: Malondialdehyde; SOD: Superoxide dismutase; ATPase: Adenosine triphosphatease; GPx: Glutathione peroxidase; Cyt-c: Cytochrome c; Akt: Protein kinase B; SD: Standard deviation. 


\section{Competing interests}

The authors declare that they have no competing interests.

\section{Authors' contributions}

QD designed the study, participated in the laboratory investigations and the design of the protocol, and wrote the manuscript. CW carried out the Western blotting, participated in the laboratory investigations, and performed the statistical analysis and helped draft the manuscript. NZ and $G L$ participated in the laboratory investigations and wrote the manuscript $M Z, Q Z, H Z$, and LL participated in analysis and interpretation. JZ directed the study. All authors have read and approved the final version of the manuscript.

\section{Acknowledgements}

This study was supported by grants from the Nature Science Foundation of Hebei Province, China (no. H2014307002).

\section{Author details}

'Department of Pharmacology, Hebei Medical University, 361 Zhongshan Eastern Road, Shijiazhuang 050017, China. ${ }^{2}$ Intensive Care Unit, Hebei General Hospital, 348 Heping Western Road, Shijiazhuang 050051, China. ${ }^{3}$ Clinical research center, Hebei General Hospital, 348 Heping Western Road, Shijiazhuang 050051, China. ${ }^{4}$ Department of Pharmacology, Children's Hospital of Hebei Province, 133 Jianhua Southern Avenue, Shijiazhuang 050031, China. ${ }^{5}$ Department of Pharmacology, Hebei Centers for Disease Control and Prevention, 97 Huaian Eastern Road, Shijiazhuang 050021, China. ${ }^{6}$ Department of chest surgery, Third Hospital of Hebei Medical University, 139 Ziqiang Road, Shijiazhuang 050051, China.

Received: 14 May 2014 Accepted: 11 December 2014 Published: 15 December 2014

\section{References}

1. Ware LB, Matthay MA: The acute respiratory distress syndrome. $N$ Engl J Med 2000, 342:1334-1349.

2. Faller S, Zimmermann KK, Strosing KM: Inhaled hydrogen sulfide protects against lipopolysaccharide-induced acute lung injury in mice. Med Gas Res 2012, 2:2-6.

3. Fried E, Weissman C, Sprung C: Postoperative sepsis. Curr Opin Crit Care 2011, 17:396-401.

4. Huang XL, Ma HJ, Zhou XH: Effect of exogenous hydrogen sulfide on polymorphonuclear neutrophil accumulation in acute lung injury rat induced by lipopolysaccharides and its mechanism. Chin J Appl Physiol 2010, 26:477-480.

5. Fujita M, Kuwano K, Kunitake R, Haqimoto N, Miyazaki H, Kawasaki M, Kawasaki M, Maeyama T, Hara N: Endothelial cell apoptosis in lipopolysaccharide-induced lung injury in mice. Int Arch Allergy Immunol 1998, 117:202-208.

6. Liaudet L, Rosenblatt-Velin N, Pacher: Role of peroxynitrite in the cardiovascular dysfunction of septic shock. Curr Vasc Pharmacol 2013, 11:196-207.

7. Fielhaber JA, Carroll SF, Dydensborg AB, Shourian M, Triantafillopoulos A, Harel S, Hussain SN, Bouchard M, Qureshi ST, Kristof AS: Inhibition of Mammalian Target of Rapamycin Augments Lipopolysaccharide-Induced Lung Injury and Apoptosis. J Immunol 2012, 188:4535-4542.

8. Liu G, Cheresh P, Kamp DW: Molecular basis of asbestos-induced lung disease. Annu Rev Patho 2013, 24:161-187.

9. Ufnal M, Żera $T$ : The role of nitric oxide, hydrogen sulfide and carbon monoxide in the regulation of the circulatory system and their pharmacotherapeutic potential. Kardiol Pol 2010, 68(Suppl 5):S436-\$440.

10. Lowicka $\mathrm{E}$, BeLtowski J: Hydrogen sulfide $\left(\mathrm{H}_{2} \mathrm{~S}\right)$ - the third gas of interest for pharmacologists. Pharmacol Rep 2007, 59:4-24.

11. Wallace $J$ L, Ferraz JG, Muscara MN: Hydrogen sulfide: an endogenous mediator of resolution of inflammation and injury. Antioxid Redox Signal 2012, 17:58-67.

12. Yu YP, Chin XL, Liu L: A Hypothesis: Hydrogen Sulfide Might Be Neuroprotective against Subarachnoid Hemorrhage Induced Brain Injury. Sci World J 2014, 2014:432318.

13. Zivkovic V, Jakovljevic V, Pechanova O, Srejovic I, Joksimovic J, Selakovic D, Barudzic N, Djuric DM: Effects of DL-homocysteine thiolactone on cardiac contractility, coronary flow, and oxidative stress markers in the isolated rat heart: the role of different gasotransmitters. Biomed Res Int 2013, 2013:318-471.

14. Ang AD, Rivers-Auty J, Hegde A, Ishii I, Bhatia M: The effect of CSE gene deletion in caerulein-induced acute pancreatitis in the mouse. Am J Physiol Gastrointest Liver Physiol 2013, 305:G712-G721.

15. Shanmugam MK, Bhatia M: The role of pro-inflammatory molecules and pharmacological agents in acute pancreatitis and sepsis. Inflamm Allergy Drug Targets 2010, 9:20-31.

16. Biermann J, Lagreze WA, Schallner N, Schwer Cl, Goebel U: Inhalative preconditioning with hydrogen sulfide attenuated apoptosis after retinal ischemia/reperfusion injury. Mol Vis 2011, 17:1275-1286.

17. Faller S, Ryter SW, Choi AM, Loop T, Schmidt R, Hoetzel A: Inhaled hydrogen sulfide protects against ventilator-induced lung injury. Anesthesiology 2010, 113:104-115.

18. Wang C, Wang HY, Liu ZW, Fu Y, Zhao B: Effect of endogenous hydrogen sulfide on oxidative stress in oleic acid-induced acute lung injury in rats. Chin Med J (Engl) 2011, 124:3476-3480.

19. Faller S, Spassov SG, Zimmermann KK, Ryter SW, Buerkle H, Loop T, Schmidt R, Strosing KM, Hoetzel A: Hydrogen sulfide prevents hyperoxia-induced lung injury by downregulating reactive oxygen species formation and angiopoietin-2 release. Curr Pharm Des 2013, 19:2715-2721.

20. Otulakowski G, Kavanagh BP: Hydrogen Sulfide in Lung Injury Therapeutic Hope from a Toxic Gas? Anesthesiology 2010, 113:4-6.

21. Bhatia M: Role of hydrogen sulfide in the pathology of inflammation. Scientifica (Cairo) 2012, 2012:159680.

22. Smith Kendra M, Mrozek Jeanne D, Simonton Susan C, Bing Dennis R, Meyers Pat A, Connett John E, Mammel MC: Prolonged partical liquid ventilation using conventional and high frequency ventilatory techniques: gas exchange and lung pathology in an animal model of respiratory distress syndrome. Crit Care Med 1997, 25:1888-1897.

23. Szapiel SV, Elson NA, Fulmer JD, Hunninghake GW, Crystal RG: Bleomycin-induced interstitial pulmonary disease in the nude, athymic mouse. Am Rev Respir Dis 1979, 120:893-899.

24. Siriporn C: Chattipakorn, Savitree Thummasorn, Jantira Sanit, Nipon Chattipakorn: Phosphodiesterase-3 inhibitor (cilostazol) attenuates oxidative stress-induced mitochondrial dysfunction in the heart. J Geriatr Cardiol 2014, 11:151-157

25. Ruiz-Meana M, Garcia-Dorado D, Miró-Casas E, Abellán A, Soler-Soler J: Mitochondrial $\mathrm{Ca}^{2+}$ uptake during simulated ischemia does not affect permeability transition pore opening upon simulated reperfusion. Cardiovasc Res 2006, 71:715-724.

26. The Acute Respiratory Distress Syndrome Network: Ventilation with lower tidal volumes as compared with traditional tidal volumes for acute lung injury and the acute respiratory distress syndrome. N Engl J Med 2000, 342:1301-1308.

27. Wheeler AP, Bernard GR: Acute lung injury and acute respiratory distress syndrome: a clinical review. Lancet 2007, 369:1553-1564.

28. Villar J, Blanco J, Añón JM, Santos-Bouza A, Blanch L, Ambrós A, Mosteiro F Basaldúa S, Fernández RL, Kacmarek RM, ALIEN Network: The ALIEN study: incidence and outcome of acute respiratory distress syndrome in the era of lung protective ventilation. Intensive Care Med 2011, 37:1932-1941.

29. Zhang H, Slutsky AS, Vincent $J$ : Oxygen free radicals in ARDS, septic shock and organ dysfunction. Intensive Care Med 2000, 26:474-476.

30. Choi JS, Lee HS, Seo KH, Na JO, Kim YH, Uh ST, Park CS, Oh MH, Lee SH, Kim YT: The effect of post-treatment $\mathrm{N}$-acetylcysteine in LPS-induced acute lung injury of rats. Tuberc Respir Dis (Seoul) 2012, 73:22-31.

31. Calfee CS, Matthay MA: Nonventilatory treatments for acute lung injury and ARDS. Chest 2007, 131:913-920.

32. O'Grady NP, Preas HL, Pugin J, Fiuza C, Tropea M, Reda D, Banks SM, Suffredini AF: Local inflammatory responses following bronchial endotoxin instillation in humans. Am J Respir Crit Care Med 2001 163:1591-1598.

33. Ruchko MV, Gorodnya OM, Zuleta A, Pastukh VM, Gillespie MN: The DNA glycosylase Ogg1 defends against oxidant-induced mtDNA damage and apoptosis in pulmonary artery endothelial cells. Free Radic Biol Med 2011, 50:1107-1113.

34. Galluzzi L, Kepp O, Kroemer G: Mitochondria: master regulators of danger signaling. Nat Rev Mol Cell Biol 2012, 13:780-788.

35. Osellame LD, Blacker TS, Duchen MR: Cellular and molecular mechanisms of mitochondrial function. Best Pract Res Clin Endocrinol Metab 2012, 26:711-723 
36. Singer $\mathrm{M}$ : The role of mitochondrial dysfunction in sepsis-induced multi-organ failure. Virulence 2014, 5:66-72.

37. Dogan SA, Trifunovic A: Modelling mitochondrial dysfunction in mice. Physiol Res 2011, 60:561-70.

38. Trentadue R, Fiore F, Massaro F, Papa F, luso A, Scacco S, Santacroce L, Brienza N: Induction of mitochondrial dysfunction and oxidative stress in human fibroblast cultures exposed to serum from septic patients. Life Sci 2012, 91:237-243.

39. Chouteau JM, Obiako B, Gorodnya OM, Pastukh VM, Ruchko MV, Wright AJ, Wilson GL, Gillespie MN: Mitochondrial DNA integrity may be a determinant of endothelial barrier properties in oxidant-challenged rat lungs. Am J Physiol Lung Cell Mol Physiol 2011, 301:L892-898.

40. Lundy CT, Shield JP, Kivvitingen EA, Vinorum OJ, Trimble ER, Morris AA: Acute respiratory distress syndrome in long-chain 3-hydroxyacyl-CoA dehydrogenase and mitochondrial trifunctional protein deficiencies. $J$ Inherit Metab Dis 2003, 26:537-541.

41. Yin F, Sancheti H, Cadenas E: Mitochondrial thiols in the regulation of cell death pathways. Antioxid Redox Signal 2012, 17:1714-1727.

42. Gu ZY, Ling YL, Xu XH, Meng AH, Li SJ: Effect of peroxynitrite on the reactivity of rabbit pulmonary arteriesin vitro. Acta PhysiolSc 2003, 55:469-474

43. Liu S, Chen JF: Strategies for therapeutic hypometabothermia. J Exp Stroke Transl Med 2012, 5:31-42.

44. Spiller F, Orrico MIL, Nascimento DC, Czaikoski PG, Souto FO, Alves-Filho JC, Freitas A, Carlos D, Montenegro MF, Neto AF, Ferreira SH, Rossi MA, Hothersall JS, Assreuy J, Cunha FQ: Hydrogen sulfide improves neutrophil migration and survival in sepsis via $\mathrm{K}^{+} \mathrm{ATP}$ channel activation. Am J Respir Crit Care Med 2010, 182:360-368.

45. Tokuda K, Kida K, Marutani E, Crimi E, Bougaki M, Khatri A, Kimura H, Ichinose F: Inhaled hydrogen sulfide prevents endotoxin-induced systemic inflammation and improves survival by altering sulfide metabolism in mice. Antioxid Redox Signal 2012, 17:11-21.

46. Aslami H, Pulskens WP, Kuipers MT, Bos AP, van Kuilenburg ABP, Wanders RJ, Roelofsen J, Roelofs JJ, Kerindongo RP, Beurskens CJ, Schultz MJ, Kulik W, Weber NC, Juffermans NP: Hydrogen sulfide donor NaHS reduces organ injury in a rat model of pneumococcal pneumosepsis, associated with improved bio-energetic status. PLoS One 2013, 8:e63497.

47. Zhang H, Zhi L, Moore PK, Bhatia M: Role of hydrogen sulfide in cecal ligation and puncture-induced sepsis in the mouse. Am J Physiol Lung Cell Mol Physiol 2006, 290:L1193-L1201.

48. Zhang H, Hegde A, Ng SW, Adhikari S, Moochhala SM, Bhatia M: Hydrogen sulfide up-regulates substance $P$ in polymicrobial sepsis-associated lung injury. J Immunol 2007, 179:4153-4160.

49. Madurga A, Mižíková I, Ruiz-Camp J, Vadász I, Herold S, Mayer K, Fehrenbach H, Seeger W, Morty RE: Systemic hydrogen sulfide administration partially restores normal alveolarization in an experimental animal model of bronchopulmonary dysplasia. Am J Physiol Lung Cell Mol Physiol 2014, 306:L684-97.

doi:10.1186/1471-2253-14-117

Cite this article as: Du et al:: In vivo study of the effects of exogenous hydrogen sulfide on lung mitochondria in acute lung injury in rats. $B M C$ Anesthesiology 2014 14:117.

\section{Submit your next manuscript to BioMed Central and take full advantage of:}

- Convenient online submission

- Thorough peer review

- No space constraints or color figure charges

- Immediate publication on acceptance

- Inclusion in PubMed, CAS, Scopus and Google Scholar

- Research which is freely available for redistribution 